performed at a different center, treated mice for 31 days, and included a comparator group of mice treated with an antibody to tumor necrosis factor. Paws were monitored regularly for signs of inflammation, and knees (in mice) or paws (in rats) were evaluated histologically at completion of all studies.

CP-690550 treatment was associated with significant, dose-dependent decreases in clinical signs of arthritis in both animal models; the highest dose $(15 \mathrm{mg} / \mathrm{kg}$ daily) resulted in a $>90 \%$ reduction in paw swelling compared with controls. Furthermore, histologic analysis showed less inflammatory cell influx and joint damage in CP-690550-treated animals than in controls; there was no difference in the histologic evidence of joint damage between mice with $\mathrm{CIA}$ that received the highest dose of CP-690550 and mice without CIA.

The authors conclude that CP-690550 effectively reduces symptoms of arthritic synovitis in these two animal models; JAK3 inhibition might, therefore, represent a novel treatment strategy for rheumatoid arthritis.

Original article Milici AJ et al. (2008) Cartilage preservation by inhibition of Janus kinase 3 in two rodent models of rheumatoid arthritis. Arthritis Res Ther 10: R14

\section{Endomorphin 1 has anti-inflammatory effects in RA and $O A$}

Endogenous opioids are thought to reduce inflammation as well as pain, but their roles in inflammatory conditions are poorly characterized. Anti-inflammatory effects of opioids could be exploited for therapeutic purposes in osteoarthritis (OA) and rheumatoid arthritis (RA), so Straub and colleagues investigated the as-yet-unknown roles of two endogenous opioids-endomorphins 1 and 2-in these two diseases.

The researchers evaluated the expression of endomorphins 1 and 2, and their effects on proinflammatory cytokines interleukin (IL)-6 and IL-8, in synovial tissue from 10 patients with RA and 10 patients with $\mathrm{OA}$ who had undergone knee replacement surgery. Both endomorphins were expressed in $\mathrm{OA}$ and RA tissues, especially in highly inflamed areas such as vessel walls and the lining layer; furthermore, cells expressing these endomorphins were macrophages, T cells and fibroblasts-cells involved in inflammation. Superfusion with endomorphins 1 and 2 had anti-inflammatory effects; endomorphin 1 inhibited secretion of IL-8 in both OA and RA tissue, and of IL-6 in RA tissue. Endomorphin 2 weakly inhibited IL-8 secretion in RA tissue but not in OA tissue, and had no effect on IL-6 secretion. In rats with adjuvant-induced polyarthritis, expression of endomorphin 1, and to a lesser extent endomorphin 2, was upregulated in synovial tissue and lymphoid organs. Importantly, endomorphin 1 injections administered to these rats attenuated inflammation in vivo.

Endomorphins are potential therapeutic agents for RA and OA, perhaps particularly for the treatment of acute episodes when inflammation has a considerable neurogenic contribution.

Original article Straub RH et al. (2008) Antiinflammatory role of endomorphins in osteoarthritis, rheumatoid arthritis, and adjuvant-induced polyarthritis. Arthritis Rheum 58: $456-466$

\section{Cardiac MRI detects subclinical right ventricular impairment in patients with SSC}

Myocardial fibrosis is thought to be common in patients with systemic sclerosis (SSc), although cardiac impairment is clinically detectable in only $20-25 \%$ of cases. Overt myocardial involvement correlates with poor prognosis in patients with SSc; however, early detection and appropriate treatment of myocardial disease might improve the outlook for affected patients. Bezante and colleagues assessed cardiac functioning in patients with SSc who had shown no signs or symptoms of cardiovascular disease.

Of 119 consecutive patients with SSc seen at a single institution, 50 patients (mean age $53.3 \pm 12.9$ years, 45 women) who had no symptoms of any serious diseases apart from SSc (limited cutaneous SSc [ $n=33$ ] or diffuse SSc $[n=17])$ were included in the study. The control group comprised 31 healthy individuals, matched for sex, age and body surface area. Both groups underwent several diagnostic tests, including Doppler echocardiography, ultrasonography and cardiac MRI.

Cardiac MRI was used to measure ejection fractions, end-diastolic volumes and end-systolic volumes of both ventricles. The data, which were adjusted for age and body 